\title{
Study of the Effects of Lower Limb Muscle Strengthening Exercises on Hospitalized Pregnant Women on Bed Rest for Prevention of Miscarriage
}

\author{
Yu Yang*, Yanzhen Rao*, Ke Sun\#, Qiuying Lin, Ying Feng, Xiaoyan Chen \\ Department of Obstetrics, The Third Affiliated Hospital of Sun Yat-sen University, Guangzhou, China \\ Email: ${ }^{\# 294597586 @ q q . c o m ~}$
}

How to cite this paper: Yang, Y., Rao, Y.Z., Sun, K., Lin, Q.Y., Feng, Y. and Chen, X.Y. (2020) Study of the Effects of Lower Limb Muscle Strengthening Exercises on Hospitalized Pregnant Women on Bed Rest for Prevention of Miscarriage. Open Journal of Nursing, 10, 1241-1250.

https://doi.org/10.4236/ojn.2020.1012090

Received: November 27, 2020

Accepted: December 28, 2020

Published: December 31, 2020

Copyright $\odot 2020$ by author(s) and Scientific Research Publishing Inc. This work is licensed under the Creative Commons Attribution International License (CC BY 4.0).

http://creativecommons.org/licenses/by/4.0/

(c) (i) Open Access

\begin{abstract}
Objective: Against a background of the two-child policy in China, the objective is to explore the effects of lower limb strengthening exercises on hospitalized pregnant women put on bed rest for prevention of miscarriage. Method: Sixty cases of pregnant women admitted to the Department of Obstetrics in one of the highest ranking hospitals in Guangzhou, China, during the period from November 2018 to December 2019 for the purpose of preventing miscarriage were selected. They were divided into an experimental group and a control group of 30 cases each. The control group was put under routine care while the intervention group conducted lower limb muscle strengthening exercise on top of the routine care. After the intervention, the conditions of lower limbs, the psychological states and the results of pregnancy for the two groups were compared. Results: After the intervention, the scores of the experimental group in anxiety, depression and postpartum depression were lower and leg circumferences were smaller compared to the control group. The difference is statistically significant $(P<0.05)$. The experimental group had longer gestation than the control group and was able to get out of bed earlier than the control group after delivery, with statistically significant difference $(P<0.05)$. Conclusion: Lower limb muscle strengthening exercises can effectively improve the physiological and psychological states of pregnant women hospitalized for prevention of miscarriage and promote their postpartum recovery.
\end{abstract}

\section{Keywords}

Prenatal Care, Exercise, Bed Rest, Preterm Birth, Psychological State

*They both are the first author of this article. 


\section{Introduction}

Since the implementation of universal two-child policy and along with the development of assisted reproductive technologies, the high-risk pregnancy population is expanding year by year [1]. As female fertility gradually declines, the incidence rates of pregnancy complications and adverse pregnancy results are continuously on the rise [2] [3]. Research data indicate that the incidence rate of high-risk pregnancy is approximately $6 \%-33 \%$ [4]. Due to the different types of complications which include threatened miscarriage, premature rupture of membranes, and preterm labor, pregnant women usually require bed rest. As a common way of clinical pregnancy management, prenatal bed rest is prescribed by as much as $95 \%$ of obstetricians to limit the activities of pregnant women [5]. Prolonged bed rest can bring serious damages to human bodies, including skeletal muscle atrophy and weakness, and thromboembolic disorders [6]. Among them, lower limb muscle atrophy is one of the most common complications. Lower limb muscle strength is one of the crucial factors that determine whether an individual can maintain independent living [7], and it affects the progress of labor to a certain extent [8]. Apart from putting a physiological burden on pregnant women who are trying to prevent miscarriage, prolonged bed rest also results in the existence of various degrees of anxiety and depression in pregnant women [9]. ACOG's publication, "Exercise during Pregnancy and Postpartum Period", recommends that appropriate exercises should be conducted during pregnancy [10]. Research also demonstrated that exercises can reduce anxiety and depression in pregnancy and effectively preserve the strength required for labor, shorten labor duration, and reduce the rate of Cesarean sections [11]. However, current studies on functional exercises during pregnancy have focused on normal pregnant women or high-risk pregnant women without restriction of activities, with a lack of functional exercises suitable for pregnant women put to long-term bed rest for prevention of miscarriage. Based on past research on functional exercises, this study has designed a set of prenatal lower limb muscle strengthening exercises for the purpose of maintaining the lower limb functions of pregnant women on long-term bed rest for prevention of miscarriage, preventing deep vein thrombosis in lower limbs, and improving the psychological state of pregnant women.

\section{Data and Method}

\subsection{General Data}

Sixty cases of pregnant women admitted to our hospital during the period from November 2018 to December 2019 were selected. Using convenience sampling, they were divided into a control group and an intervention group according to their order sequence of admission to the hospital, with 30 cases in each group. The standards of inclusion were as follows: gestation at the time of admission to the hospital $\leq 28$ weeks; expected period of hospitalization $>28$ days; condition being stable after treatment with medication; having been assessed by a specialist 
to be fit for conducting exercises; and informed consent given willingly to participate in this study. Standards of exclusion were: pregnant women with a history of abnormal lower limb muscle strength; condition being unstable; unfit for exercises, and having a past history of mental disorder, mental retardation; or major organic disease. The difference in comparison of general demographic data of the two groups is insignificant $(P>0.05)$.

\subsection{Method}

The control group was given routine prenatal care. Support stockings were worn on both lower limbs for 10 hours a day; air compression therapy for both lower limbs was given twice a day for half an hour in each session for four weeks. A uniform brand (Maiershu, model AF) of support stockings was used, and suitable models were chosen based on leg circumferences. The intervention group, on top of receiving the routine care, conducted functional prenatal lower limb muscle strengthening exercises designed by the hospital as functional exercises.

1) An Intervention Team for the prenatal lower limb muscle strengthening exercises was set up, comprising of a chief physician, a chief nursing officer, an attending physician, two supervisor nurses, and a nurse. Specifically, they were in charge of guiding the design of the fitness exercises, explanation to patients, and demonstration of the intervention method. Members of the team had all undergone uniform educational training.

2) Assessment of patients: Assessment before exercise: Condition of the pregnant women on bed rest, no contractions; no discomfort of chest tightness or shortness of breath; and normal fetal heart sounds and fetal movement. Assessment during exercise: monitoring whether the pregnant women had any complaints of discomfort, or whether there were abnormalities of vaginal bleeding and uterine contractions. Once abnormality was found, exercises were terminated immediately and the appropriate treatments were given. Monitoring after exercise: monitoring of fetal heart sounds and the pregnant women's blood pressure and pulses. In case of fetal heart abnormality, oxygen would be given, with change of posture. If the vital signs of a pregnant woman were found to be unstable, close monitoring would be given. If the pregnant woman failed to recover, a physician would be notified for further actions.

3) Design of prenatal lower limb muscle strengthening exercises

The prenatal lower limb muscle strengthening exercises were designed according to physiological characteristics during pregnancy and matters for attention during bed rest for prevention of miscarriage, with reference to training methods for bed-rest patients and multidisciplinary rehabilitation as well as published literature in China and overseas. Through literature review, consultation with experts, and clinical experience, a set of prenatal lower limb muscle strengthening exercises was designed by selecting the best. Each movement had been pre-tested clinically for its exercise time, intensity, and purpose in five cases by the pregnant women in person, and their opinions were taken into account. 
Four movements were eventually confirmed. On top of the routine care given, lower-limb muscle strengthening exercises were added. The lower limb muscle strengthening exercises were designed through observing the principle of orderly progress in exercises and the principle of working from bottom to top in lower limb exercise physiology. The specific program is as follows:

Preparatory pose: The pregnant woman lies on her back, relaxing the whole body and stretches her lower limbs.

a) Ankle pumps exercise:

i) Ankle joint dorsiflexion and plantarflexion exercise: Slowly bend the toe side of feet inward, pointing to self as much as possible, and hold at the maximum position for 10 seconds. Then slowly press the toe side of feet downward and hold at the maximum position for 10 seconds. Repeat back and forth 10 times. ii) Ankle rotation exercise: centered on the ankle and rotate the toes 360 degrees, maintaining the maximum extent of movement; repeat 20 times.

b) Heel exercise:

Bend the knees of both lower limbs, with bottom of the feet against the bed. Move the heel slowly along the surface of bed toward the buttock, and then straighten slowly. Alternate the lower limbs for the above movement.

c) Straight leg raise exercise:

Keeping the knee straight for the lower limb on one side, raise the leg at an angle of at least 15 degrees and hold for at least 10 seconds. After raising the lower limb 10 times consecutively, switch to the lower limb on the other side.

4) Training method

The lower limb muscle strengthening exercises were recorded as an MP4 format video. The first part was a one-minute presentation of the functions of the strengthening exercises and matters for attention. The second part was a 15-minute video of movements for learning. The background music was selected from Mozart's Serenade. The video file was saved in a USB flash drive. The LCD flat screen televisions in the wards were confirmed to be operating normally to ensure that patients can view the TV screens. The video was played to the experimental group after breakfast, lunch, and dinner on daily basis. Trained nurses provided guidance and made assessments on-site according to the conditions of the patients.

\subsection{Assessment Method}

\subsubsection{Assessment Tool}

Assessments were made when the pregnant woman was admitted to the hospital and four weeks after the intervention by using the Self-Rating Anxiety Scale (SAS) and the Self-Rating Depression Scale (SDS). One week after delivery, the Edinburgh Postpartum Depression Scale was used to make assessment.

\subsubsection{Observational Indicators}

The pregnant women's thigh and calf circumferences, lower limb strength, weeks of gestation, modes of delivery, time taken to be out of bed for postpartum 
activity, and postpartum complications were observed and recorded. The assessment standards of some of the indicators were as follows:

1) Lower limb strength

Assessment was made according to the six-grade muscle strength standard. A grade-five assessment indicates normal muscle strength while others were abnormal strength.

2) Others

Time taken to be out of bed for postpartum activity refers to getting to the state when a woman can stand with both feet on the ground for three minutes or more after giving birth. Postpartum complications refer to whether there was fever within one week from the delivery and whether there was postpartum bleeding within 42 days after giving birth.

\subsection{Statistical Method}

SPSS 20.0 software is used for statistical analysis. Percentages, average value \pm standard deviation, and median expression were used in statistical computation. The methods used were t-test, chi-square test, Fisher exact probability test, and nonparametric Mann-Whitney $\mathrm{U}$ test, with $P<0.05$ indicating a significant difference.

\section{Results}

1) Before the intervention, no statistically significant difference was found in anxiety and depression when comparing the two groups $(P>0.05)$. After the intervention, anxiety and depression of the experimental group were both lower than the control group, with statistically significant difference $(P<0.05)$, as shown in Table 1.

2) Before the intervention, no statistically significant difference was found in leg circumferences and muscle strength when comparing the two groups. After the intervention, the calf circumferences of both left and right legs of the experimental group were larger than the control group, with statistically significant difference $(P<0.05)$, as shown in Table 2.

3) No statistically significant difference was found in modes of delivery and postpartum complications when comparing the two groups (both $P>0.05$ ). Gestation period of the experimental group was longer than the control group $(P$

Table 1. Comparison of scores of anxiety and depression before and after intervention.

\begin{tabular}{cccccc}
\hline \multirow{2}{*}{ Group } & $\boldsymbol{n}$ & \multicolumn{2}{c}{ Anxiety } & \multicolumn{2}{c}{ Depression } \\
\cline { 3 - 6 } & & Before intervention & After intervention & Before intervention & After intervention \\
\hline Experimental & 30 & $37.00(32.75,42.00)$ & $30.00(26.00,32.00)$ & $39(36,48)$ & $32(28,37)$ \\
Control & 30 & $36.00(33.00,41.00)$ & $32.00(28.00,36.00)$ & $39(37,44)$ & $34(32,40)$ \\
$Z$ Value & & -0.432 & -10.262 & -0.753 & -7.457 \\
$P$ Value & & $>0.05$ & $<0.05$ & $>0.05$ & $<0.05$ \\
\hline
\end{tabular}


Table 2. Comparison of leg circumference and muscle force after intervention.

\begin{tabular}{|c|c|c|c|c|c|c|c|}
\hline \multirow{2}{*}{ Group } & \multirow{2}{*}{$n$} & \multicolumn{4}{|c|}{ Leg circumference $(\mathrm{cm}, \mathrm{x} \pm \mathrm{s})$} & \multicolumn{2}{|c|}{ muscle strength } \\
\hline & & Left thigh & Left calf & Right thigh & Right calf & normal & abnormal \\
\hline Experimental & 30 & $40.15 \pm 3.66$ & $31.39 \pm 3.56$ & $39.63 \pm 5.26$ & $31.39 \pm 3.65$ & 22 & 8 \\
\hline Control & 30 & $40.05 \pm 3.98$ & $29.30 \pm 3.85$ & $40.16 \pm 4.00$ & $29.25 \pm 3.05$ & 18 & 12 \\
\hline Statistics & & $t=0.095$ & $t=2.192$ & $t=0.095$ & $t=2.484$ & \multicolumn{2}{|c|}{$\chi^{2}=0.412$} \\
\hline$P$ Value & & $>0.05$ & $<0.05$ & $>0.05$ & $<0.05$ & \multicolumn{2}{|c|}{$>0.05$} \\
\hline
\end{tabular}

Table 3. Comparison of pregnancy outcomes between two groups.

\begin{tabular}{|c|c|c|c|c|c|c|c|}
\hline \multirow{2}{*}{ Group } & \multirow{2}{*}{$n$} & \multicolumn{2}{|c|}{ delivery mode [cases (\%)] } & \multirow{2}{*}{$\begin{array}{l}\text { Gestation period } \\
\text { [weeks, M (P25, } \\
\text { P75)] }\end{array}$} & \multirow{2}{*}{$\begin{array}{l}\text { Postpartum } \\
\text { complications } \\
\text { [cases (\%)] }\end{array}$} & \multirow{2}{*}{$\begin{array}{l}\text { Postpartum activity } \\
\text { time [hour, } M \\
\quad(P 25, P 75)]\end{array}$} & \multirow{2}{*}{$\begin{array}{c}\text { Postpartum } \\
\text { depression score } \\
\text { [score, } M(P 25, P 75)]\end{array}$} \\
\hline & & vaginal delivery & cesarean delivery & & & & \\
\hline Experimental & 30 & $18(60.0)$ & $12(40.0)$ & $37.00(36.00,37.00)$ & $6(20.0)$ & $30(25,35)$ & $9(8,10)$ \\
\hline Control & 30 & $13(43.3)$ & $17(56.7)$ & $36.57(35.71,37.00)$ & $9(30.0)$ & $32(25,36)$ & $9(9,11)$ \\
\hline Statistics & & \multicolumn{2}{|c|}{$\chi^{2}=0.301$} & $Z=-7.620$ & $\chi^{2}=0.552$ & $Z=-2.670$ & $Z=-3.425$ \\
\hline$P$ Value & & \multicolumn{2}{|c|}{$>0.05$} & $<0.05$ & $>0.05$ & $<0.05$ & $<0.05$ \\
\hline
\end{tabular}

$<0.05)$; time taken to be out of bed for postpartum activity was less than the control group $(P<0.05)$; and postpartum depression score was lower than the control group, as shown in Table 3.

\section{Discussions}

\subsection{Prenatal Lower Limb Muscle Strengthening Exercises Improving the Feelings of Anxiety and Depression in Pregnant Women on Bed Rest}

This study has found that, after the intervention, the scores of anxiety and depression by the experimental group were both lower than those of the control group. The study of Perales et al. [12] also proved that conducting exercises under supervision during pregnancy can reduce the occurrence of depression in pregnant women. Similarly, in a study of Kim [13] on the effects of exercises conducted in bed on the psychological symptoms of high-risk pregnant women, it was found that exercises conducted in bed did not increase the risk to fetuses for hospitalized high-risk pregnant women. At the same time, these exercises had functioned to alleviate body discomfort and anxiety. Regular amount of daily lower limb exercises at regular timing can shift the attention of pregnant women from the health of the fetuses in uterus to the control of their own limbs, thus easing their nervous feeling. Exercise intensity characterized by regulated relaxation and tightening can provide better relief and positive channeling of the negative feeling caused by long-term confinement to bed, thereby reducing anxiety and depression. At the same time, the results of this study indicate that the experimental group had an obviously lower scoring of depression compared to the control group, which is statistically significant. Regular prenatal lower limb 
exercises to combat the adverse effects of bed rest for the purpose of preventing miscarriage enable the women giving birth to positively deal with the psychological impact brought by labor pain and the changes in family structure after delivery, thus rendering her to be more emotionally stable and able to feed her newborn with a positive mind.

\subsection{Prenatal Lower Limb Muscle Strengthening Exercises Maintaining the Condition of Lower Limb Functions for Pregnant Women}

As shown in a research study [14], "dramatic change in muscle mass occurs within 4 - 6 weeks of bed rest, accompanied by decrease of $6 \%$ to $40 \%$ in muscle strength". The results of this study show that calf circumference of the experimental group is better than that of the control group, indicating that lower limb muscle strengthening exercises are able to reduce calf muscle atrophy of pregnant women on long-term bed rest. The reasons are analyzed as follows: 1) this set of muscle strengthening exercises focused on exercising the calf of lower limbs, thus preventing muscle atrophy as a result of lack of walking; 2) when changing the postures of lower limbs continuously, the isometric exercises and isotonic exercises included in this set of muscle strengthening exercises also prevented the limbs from staying at the same position, thus preserving the flexibility of joints; in a situation of long-term bed rest, 3) after four weeks' training of lower limb muscle strengthening exercise, the pregnant women's awareness of the need to exercise their lower limbs was strengthened, corresponding to the maintenance of the lower limbs' ability to exercise.

\subsection{Prenatal Lower Limb Muscle Strengthening Exercises Lengthening Gestation and Reducing Incidence of Preterm Birth}

Preterm birth is a social issue that affects the health of the population. As indicated by the Global Report on Preterm Birth in May, 2012, the incidence rate of global preterm birth is $>10 \%$. In China, the incidence rate of preterm birth is around $7 \%$, and preterm birth has become the number one cause of infant mortality in China. Concurrently, premature infants constitute a high-risk group in terms of slow growth, infectious disease and lagging behind in development in both infancy and childhood. The proportions of premature infants suffering from diseases such as cerebral palsy and chronic lung disease are evidently higher than full-term infants. As shown in the results of this study, gestation of the experimental group was evidently longer than that of the control group, with statistically significant difference. After four weeks of regular intervention, the condition of the pregnant women confined to bed rest for prevention of miscarriage had transited from the initial feeling of aversion to general acceptance and were more willing to cooperate in treatments given by the medical staff. Appropriate exercises will stimulate the development of brain, sensory organs and neurological system of a fetus in the uterus, and at the same time improve blood 
circulation of the lower limbs, thereby resulting in improved perfusion to organs, including the placenta, throughout the body, positive impact on the intrauterine environment, and promotion of metabolism of the fetus in the uterus. As a result, gestation can be lengthened and the incidence of preterm birth reduced.

\subsection{Prenatal Lower Limb Muscle Strengthening Exercises Shortening the Time Taken to be out of Bed for Postpartum Activity and Speeding up Postpartum Recovery}

As shown by the results of this study, the time taken for the experimental group to be out of bed for postpartum activity was evidently shorter than that of the control group, with statistically significant difference. Research has indicated that getting out of bed for activities early enough can effectively lower the formation of deep vein thrombosis, promote intestinal peristalsis, reduce the incidence of bowel obstruction, and raise the capability and confidence of the mother in feeding her baby [15] [16]. The lower limb muscle strengthening exercises carried out during the period of preventing miscarriage allow regular exercising of a pregnant woman's joints and muscle, facilitating the positive development of confidence in the pregnant woman and her getting out of bed to resume activities after delivery. At the same time, regular exercises during pregnancy can improve body condition, boost immunity, reduce the incidence of postpartum complications, and speed up recovery.

Along with social development and changes in living habits, high-risk pregnancy is continuously on the rise. High-risk pregnancy not only impacts the physical health and living quality of pregnant women, but also increases morbidity and mortality of new infants in the perinatal period. Appropriate and effective lower limb muscle strengthening exercises can extend gestation, reduce preterm birth, raise the muscle strength of the lower limbs of such population, and ease the feeling of anxiety during the period of preventing miscarriage, and improve the results of pregnancy. Due to the limitation of time and circumstances, it was not possible to conduct a comprehensive B-mode ultrasonic testing for the subjects, thus lacking an exploration of deep vein thrombosis in the lower limbs. During the study period, the number of pregnant women on bed rest for prevention of miscarriage that met the standards of inclusion was relatively small in number and had come from the same hospital. Hence, it is necessary to further expand the sample size for the promotion of this project.

\section{Acknowledgements}

The Scientific Research Project of Guangdong Nursing Society (gdhlxueh2019zx 337).

\section{Conflicts of Interest}

The authors declare no conflicts of interest regarding the publication of this paper. 


\section{References}

[1] Teng, X., Shane, M.I. and Pan, S. (2020) The Changing Situation about Maternal Age, Risk Factors and Pregnancy Outcomes after the Two-Child Policy: A Retrospective Cohort Study. Annals of Palliative Medicine, 9, 824-834. https://doi.org/10.21037/apm.2020.04.27

[2] Frederiksen, L.E., Ernst, A., Brix, N., et al. (2018) Risk of Adverse Pregnancy Outcomes at Advanced Maternal Age. Obstetrics \& Gynecology, 131, 457-463. https://doi.org/10.1097/AOG.0000000000002504

[3] Qin, J., Liu, X., Sheng, X., et al. (2016) Assisted Reproductive Technology and the Risk of Pregnancy-Related Complications and Adverse Pregnancy Outcomes in Singleton Pregnancies: A Meta-Analysis of Cohort Studies. Fertility and Sterility, 105, 73-85. https://doi.org/10.1016/j.fertnstert.2015.09.007

[4] Holness, N. (2018) High-Risk Pregnancy. Nursing Clinics of North America, 53, 241-251. https://doi.org/10.1016/j.cnur.2018.01.010

[5] Bigelow, C. and Stone, J. (2011) Bed Rest in Pregnancy. Mount Sinai Journal of Medicine, 78, 291-302. https://doi.org/10.1002/msj.20243

[6] Aissaoui, N., Martins, E., Mouly, S., et al. (2009) A Meta-Analysis of Bed Rest versus Early Ambulation in the Management of Pulmonary Embolism, Deep Vein Thrombosis, or Both. International Journal of Cardiology, 137, 37-41. https://doi.org/10.1016/j.ijcard.2008.06.020

[7] Nomura, T., Kawae, T., Kataoka, H. and Ikeda, Y. (2018) Assessment of Lower Extremity Muscle Mass, Muscle Strength, and Exercise Therapy in Elderly Patients with Diabetes Mellitus. Environmental Health and Preventive Medicine, 23, Article No. 20. https://doi.org/10.1186/s12199-018-0710-7

[8] Bø, K., Hilde, G., Jensen, J.S., et al. (2013) Too Tight to Give Birth? Assessment of Pelvic Floor Muscle Function in 277 Nulliparous Pregnant Women. International Urogynecology Journal, 24, 2065-2070. https://doi.org/10.1007/s00192-013-2133-8

[9] Gallagher, A., Kring, D. and Whitley, T. (2020) Effects of Yoga on Anxiety and Depression for High Risk Mothers on Hospital Bedrest. Complementary Therapies in Clinical Practice, 38, 101079. https://doi.org/10.1016/j.ctcp.2019.101079

[10] Haakstad, L.A., Torset, B. and Bø, K. (2016) What Is the Effect of Regular Group Exercise on Maternal Psychological Outcomes and Common Pregnancy Complaints? An Assessor Blinded RCT. Midwifery, 32, 81-86. https://doi.org/10.1016/j.midw.2015.10.008

[11] Davenport, M.H., Ruchat, S.-M., Sobierajski, F., et al. (2019) Impact of Prenatal Exercise on Maternal Harms, Labour and Delivery Outcomes: A Systematic Review and Meta-Analysis. British Journal of Sports Medicine, 53, 99-107. https://doi.org/10.1136/bjsports-2018-099821

[12] Perales, M., Refoyo, I., Coteron, J., et al. (2015) Exercise during Pregnancy Attenuates Prenatal Depression: A Randomized Controlled Trial. Evaluation \& the Health Professions, 38, 59-72. https://doi.org/10.1177/0163278714533566

[13] Kim, Y.J. and Park, Y.-J. (2018) Effect of Structured Bed Exercise on Uterine Contractions, Fetal Heart Rate Patterns, and Maternal Psychophysical Symptoms of Hospitalized High-Risk Pregnant Women: A Randomized Control Trial. Asian Nursing Research, 12, 1-8. https://doi.org/10.1016/j.anr.2017.12.003

[14] Bloomfield, S.A. (1997) Changes in Musculoskeletal Structure and Function with Prolonged Bed Rest. Medicine \& Science in Sports \& Exercise, 29, 197-206. https://doi.org/10.1097/00005768-199702000-00006 
[15] Bane, S.M. (2015) Postpartum Exercise and Lactation. Clinical Obstetrics and Gynecology, 58, 885-892. https://doi.org/10.1097/GRF.0000000000000143

[16] Cooper, D.B. and Yang, L. (2020) Pregnancy and Exercise. StatPearls Publishing, Treasure Island, FL. 